\title{
Uncertainties in façade fire tests - measurements and modeling
}

\author{
Johan Anderson, Lars Boström, Robert Jansson McNamee and Bojan Milovanović* \\ SP Technical Research Institute of Sweden, Fire Research, Borås, Sweden \\ * Faculty of Civil Engineering, Department of Materials, University of Zagreb, Croatia
}

\begin{abstract}
In this paper a comparison between test and modelling results are performed for two large-scale façade fire testing methods, namely SP Fire 105 and BS 8414-1. In order to be able to compare tests and modelling the uncertainties have to be quantified both in the test and the modelling. Here we present a methodology based on deterministic sampling to quantify uncertainties in the modelling input. We find, in general good agreement between the models and the test results. Moreover, temperatures estimated by plate thermometers is indicated to be less sensitive to small variations in model input and is thus suitable for these kind of comparisons.
\end{abstract}

\section{INTRODUCTION}

The SP Fire 105 [1] and the British Standards BS 8414-1:2002 [2] are two among a variety of different test methods that are used to evaluate fire spread from floor to floor via external walls for different wall claddings, insulations and geometrical considerations [3-6]. Internal fire spread in enclosed burnable components in the system as well as recording falling down of parts and the occurrence of burning droplets are also partly evaluated. The SP Fire 105 test method for façade systems was defined in 1985 [1] and simulates a three-story apartment building (height $6.7 \mathrm{~m}$, width $4 \mathrm{~m}$ and depth $1.6 \mathrm{~m}$ ) i.e. a large scale façade test method, see Fig. 1. The BS 8414-1 is similar however it includes a return wall or wing on one side. The fire exposure conditions represent a fully-developed fire in a room, venting through an opening such as a window aperture that exposes the cladding to external flames. The main differences between the methods are, firstly the fuel used and secondly the height of the combustion chamber and the fact that the British test can be performed outside. In BS 8414-1 the square opening of the combustion chamber has side length of $2 \mathrm{~m}$ and the fire source is a wood crib with a nominal total effective energy release of $4500 \mathrm{MJ}$ over 30 minutes at a peak rate of $3 \pm 0.5 \mathrm{MW}$. In SP Fire 105 the fuel consists of 601 heptane burning in a special purpose tray to stabilize the flames and the height of the combustion chamber is $710 \mathrm{~mm}$. The fuel consumed in the SP method corresponds to a total energy release of $1830 \mathrm{MJ}$ and the peak rate is approximately 2.0 MW. The SP Fire 105 method has then been presented internationally for different standardization committees and has been classified as a valid test method in Sweden, Denmark and Norway although it is not presently a European standard.

This is an Open Access article distributed under the terms of the Creative Commons Attribution License 2.0, which permits unrestricted use, distribution, and reproduction in any medium, provided the original work is properly cited. 


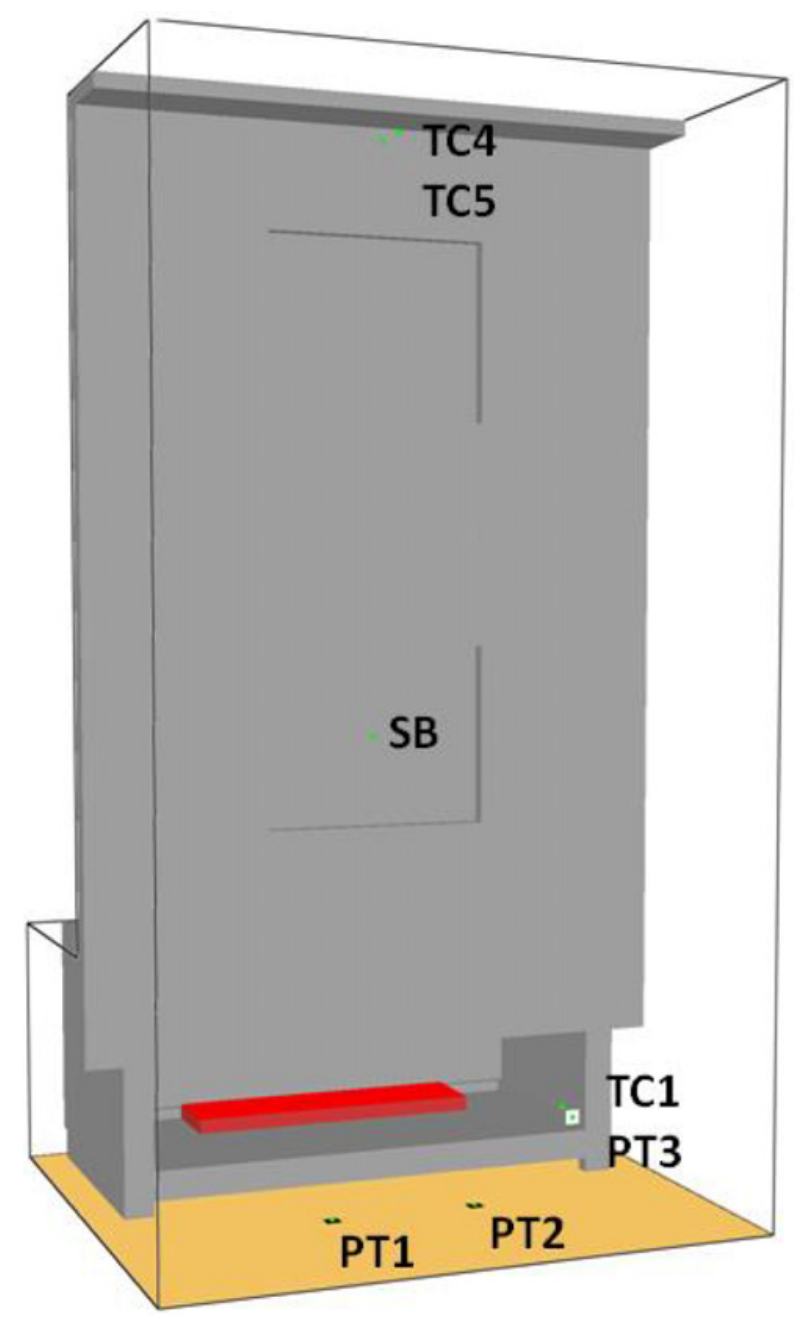

Figure 1. The model of the SP Fire 105 façade fire test.

In a previous paper by Anderson et al. [7] results regarding two series of tests according to BS 8414-1 that have been carried out outdoors in Zagreb, Croatia are presented. In each test three façade rigs were used with different test specimens, i.e. three different types of external thermal insulation composite systems (ETICS) was tested at the same time. In addition to the measurements described in the standard, temperatures were measured at different heights and in front of the combustion chamber with different types of thermocouples. In comparison, nine tests according to SP Fire 105 were carried out indoors in a fire testing hall, and thus the ambient conditions are more controlled. The nine tests presented were, four tests of combustible insulation with a protecting render, three different wood façade systems with wood impregnated with fire retardants, one test with combustible insulation (phenolic resin) protected with bricks and one where the insulation of phenolic resin was directly exposed. As in the Zagreb tests described above the thermal exposure was measured $0.5 \mathrm{~m}$ from the combustion chamber using three additional plate thermometers not specified in the test method. Both methods define an amount of fuel to be used, in BS 8141-1 a volume of wood and in SP Fire 105 a volume of heptane, as well as the geometry of the combustion chamber. It is thus not possible to regulate the heat exposure to the façade 
surface during the test, and it may be different from test to test due to factors such as air movements around the combustion chamber and geometry of the test specimens. For example the thickness of the test specimen will have an effect since energy will be absorbed by the boundaries and the flame will be mixed with more cool air before the fire reaches the façade surface.

The current paper will be divided into two parts where the first aim at quantifying the uncertainties induced by modelling and unknown conditions such as material properties and numerical input parameters. The second part will be devoted to comparisons to experiments. In this paper the previous study [7] is extended to accommodate for more analysis as well as numerical investigations. The fire dynamics is investigated by numerical means similar to what was done in several recent papers [8-10], where tests according to the test method SP Fire 105 were modelled with good correspondence between measurements and experimental findings. The numerical model was constructed in the CFD program Fire Dynamics Simulator (FDS) version 6, McGrattan, et al. (2013) [11], with analogous geometry and instrumentation as in the described fire tests. Here we present the results from a series of simulations with varying thicknesses of the façade specimens and varying thermal properties as well as a number of different fuels.

\subsection{Theoretical framework for evaluating uncertainties by deterministic sampling}

Due to the lack of knowledge of the precise details of the system, seemingly random behavior can be governed by deterministic, though non-linear models. Thus, in such systems small variations may have a significant change in the outcome. Most of the calculations both numerical and analytical are often performed with very little thought given to these uncertainties and thus designs are many times based on potentially inaccurate information. Moreover, modeling is often performed in stages by different numerical or analytical tools where the uncertainties may propagate through the system without much control $[12,13]$. It is usually a very difficult task to objectively establish the confidence levels in numerical predictions. Uncertainty Quantification (UQ) is the science of quantitative characterization and reduction of uncertainties in numerical studies and real world experimentation. UQ aims at determining how likely certain outcomes are if some aspects of the system are unknown. Deterministic Sampling (DS) [14] is a relatively new method used for Uncertainty Quantification (UQ), is employed in this paper to offer an efficient alternative. The method is based on the idea that a continuous probability density function can be replaced by an ensemble of discrete deterministic samples, provided that the two representations have the same statistical moments.

Quality assurance in CFD simulations [15] is of great importance in order to provide with reliable simulations and with the possibility of validation against experimental results. In this section we shortly summarize the theoretical background that is the basis for the explicit choices of ensembles used in this work. We will construct an example where seven parameters are modeled uncertain since this is the needed input to the numerical model however to reduce to computational time we will reduce the study to include only a few particular parameters of interest. In the absence of correlations, the ensemble of $m$ samples is given by,

$$
\Sigma=\langle\theta\rangle \otimes 1^{1 \times m}+\operatorname{diag}\left(\langle\theta\rangle \circ \operatorname{std}\left(\frac{\theta}{\langle\theta\rangle}\right)\right) \cdot \hat{V},
$$

where $1^{1 \times m}$ denotes a row vector of $m$ "ones", $\otimes$ outer product, o element-wise multiplication, and $\operatorname{diag}(\mathrm{X})$ is the diagonal matrix with the vector $\mathrm{X}$ on its diagonal. The excitation matrix $\hat{V}$ contains all variations; each column describes one "normalized" model sample variation from its mean. 


\section{MATEC Web of Conferences}

The suggested ensembles formed by the given the information above where it appears that there are $n=7$ parameters that need to be modeled uncertain. For instance the standard (STD) ensemble can be used,

$$
\begin{aligned}
\hat{V}_{S T D} & =\sqrt{n} \cdot\left(I_{n \times n}-I_{n \times n}\right) \\
& =\left(\begin{array}{ccccccccccccccc}
\sqrt{7} & 0 & 0 & 0 & 0 & 0 & 0 & -\sqrt{7} & 0 & 0 & 0 & 0 & 0 & 0 \\
0 & \sqrt{7} & 0 & 0 & 0 & 0 & 0 & 0 & -\sqrt{7} & 0 & 0 & 0 & 0 & 0 \\
0 & 0 & \sqrt{7} & 0 & 0 & 0 & 0 & 0 & 0 & -\sqrt{7} & 0 & 0 & 0 & 0 \\
0 & 0 & 0 & \sqrt{7} & 0 & 0 & 0 & 0 & 0 & 0 & -\sqrt{7} & 0 & 0 & 0 \\
0 & 0 & 0 & 0 & \sqrt{7} & 0 & 0 & 0 & 0 & 0 & 0 & -\sqrt{7} & 0 & 0 \\
0 & 0 & 0 & 0 & 0 & \sqrt{7} & 0 & 0 & 0 & 0 & 0 & 0 & -\sqrt{7} & 0 \\
0 & 0 & 0 & 0 & 0 & 0 & \sqrt{7} & 0 & 0 & 0 & 0 & 0 & 0 & -\sqrt{7}
\end{array}\right)
\end{aligned}
$$

Another example is the binary (BIN) ensemble,

$$
\hat{V}_{B I N}=\left(\begin{array}{l}
+1-1+1-1+1-1+1-1 \\
+1+1-1-1+1+1-1-1 \\
-1+1+1-1-1+1+1-1 \\
+1+1+1+1-1-1-1-1 \\
-1-1+1+1+1+1-1-1 \\
-1+1+1-1+1-1-1+1 \\
+1-1+1-1-1+1-1+1
\end{array}\right)
$$

Note: The maximum variation of the standard ensemble is $\sqrt{7}$, while it is only 1 for the binary. By varying all parameters in all samples of the binary ensemble, its maximum variation is minimized. The risk of "saturating" the model is thereby minimal with the BIN ensemble. The standard ensemble is easily generalized, but the binary ensemble has a more complex construction. Calculate the model ensemble by evaluating the model $H$ for every sample (column) of $\Sigma$, producing the row vector $H(\Sigma)$ of $\mathrm{m}$ results. The expected result is given by,

$$
\langle H\rangle=\langle H(\Sigma)\rangle=H(\Sigma) \cdot(1 / m)^{m \times 1} .
$$

Compare with $H(\langle\theta\rangle)$ (ordinary deterministic result) to have an idea about non-linear effects. If the difference is large, non-linear effects are significant. If the difference is small, it is not sure that the model can be approximated to be linear, even though it is likely.

The variance of the model result is given by,

$$
\operatorname{var}(H)=\left\langle(H(\Sigma)-\langle H(\Sigma)\rangle)^{2}\right\rangle=\left(H(\Sigma)-\langle H(\Sigma)\rangle \cdot 1^{1 \times m}\right)^{2} \cdot(1 / m)^{m \times 1} .
$$

Assuming a coverage factor $k=2$ for also the result, the modeling uncertainty will be,

$$
\operatorname{unc}(\mathrm{H})=2 \sqrt{\operatorname{var}(H)} \text {. }
$$

In summary, the confidence interval of the modeling result is given by,

$$
\lfloor\langle\mathrm{H}\rangle-2 \sqrt{\operatorname{var}(H)},\langle\mathrm{H}\rangle+2 \sqrt{\operatorname{var}(H)}\rfloor .
$$

The methodology will now be utilized to assess uncertainties in a CFD model of the SP Fire 105 façade fire test method. 
Table 1. The seven parameters to be modelled uncertain.

\begin{tabular}{|l|l|l|}
\hline Parameter & Mean & Deviation \\
\hline Radiation angles & 100 & \pm 50 \\
\hline Placement of PT3 & & $\pm 5 \mathrm{~cm}$ (one cell) \\
\hline Placement of TC1 & & $\pm 5 \mathrm{~cm}$ (one cell) \\
\hline HRR (Heat Release Rate) & Babrauskas see Fig. & $\pm 10 \%$ \\
\hline Thermal conductivity of specimen & 0.242 & $\pm 30 \%$ \\
\hline Specific heat of specimen & 1000 & $\pm 30 \%$ \\
\hline Density of specimen & 975 & $\pm 30 \%$ \\
\hline
\end{tabular}

\subsection{Estimating uncertainties in a CFD model}

In general we find good agreement and qualitatively the same behavior in comparing simulation results to the experimental data, see Ref. [7-10] however there are lot uncertainties accompanied to the input to simulations. Thus we have investigated the effects of uncertainties in seven parameters in the model [7-10] of the SP Fire 105 façade fire test of previous simulations. In Ref. [10] a grid resolution study was performed where it was concluded that a cubic grid of $5 \mathrm{~cm} \times 5 \mathrm{~cm} \times 5 \mathrm{~cm}$ is suitable for this model. Note that we will first discuss uncertainties stemming from the simulations and then compare to experimental data. The parameters with assumed uncertainties are summarized in Table 1 below, note that all uncertainties are assumed and are only used in this model to investigate how they propagate through a non-linear model. The general problem of uncertainty quantification in CFD models is usually limited by long simulation times and thus ensembles with relatively few samples/simulations however employing deterministic sampling methods we are able to perform adequate uncertainty quantification with fewer simulations. Here we can estimate the variance in the output with only eight simulations where seven parameters to be modelled uncertain. Although, the assumed variations in the material parameters are larger than can be assumed from measurements however this variation is used to assess the dependence of different material properties of the façade on the computed results. The parameters in Table 1 are given with max deviation around the mean value.

Using the parameters in combination with the BIN ensemble we will compute the variation in the output. The Heat Release Rate (HRR) as a function of time is presented in Fig. 2 as adopted from Ref. [16].

The results of the uncertainty quantification will be assessed for the thermocouples TC1 (thermocouple 1) and TC4 (Thermocouple 4), the plate thermometer PT3 and the heat flux gauge SB using the HRR shown in Fig. 2.

The results in Figs. 3 to 6 show that uncertainties can vary quite a lot depending on what output parameter is considered. The mean value of the eight simulations is used in computing the standard deviation. Here it is interesting to note that this mean values almost exactly coincides with the mean found by performing one simulation taking the mean values for all seven parameters however somewhat less fluctuations in the output is found. In Figs. 3 to 6 we present the deviations using one standard deviation. In Fig. 3, the result for TC1 (Thermocouple 1 in the lower right corner of the fire room) is displayed and variations up to approximately $10 \%$ which is of the order of the variation in HRR can be found. In the figure TC1+ and TC1 - denote the upper and lower bounds. However, it is instructive to investigate which parameter actually influence the output the most, it is found that placement mostly affects the uncertainty and to somewhat lesser degree the HRR. The uncertainty in the simulation data of the PT3 (in the lower right corner of the fire room) result is shown in Fig. 4. Here a very small variation is found, around 3\% significantly smaller than the variation in HRR. Note that in this position the simulation significantly over predicts the temperature whereas the comparative TC values are rather well represented, see Ref. [10]. We find that these additional uncertainties cannot explain the excess simulated temperature data. One possible reason for the discrepancies is the flame suppressing grid on the fire source and that may change the local dynamics compared to that of burning from a liquid surface. 


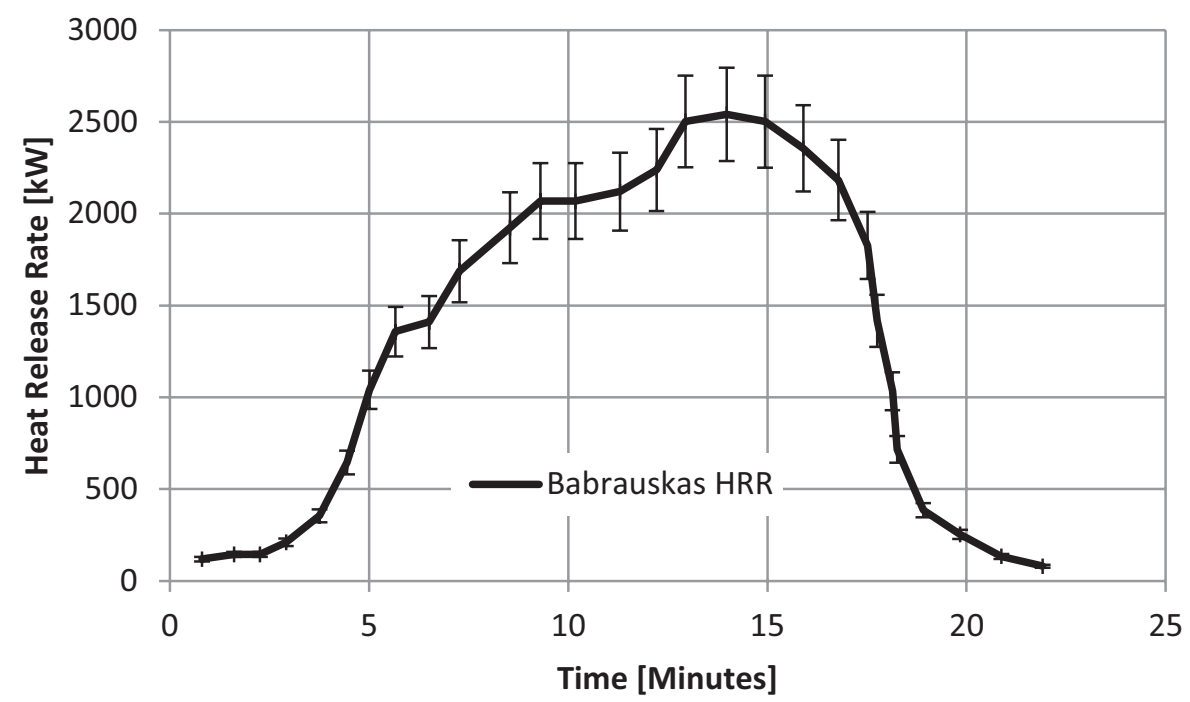

Figure 2. The heat release rate as a function of time with uncertainty bars.

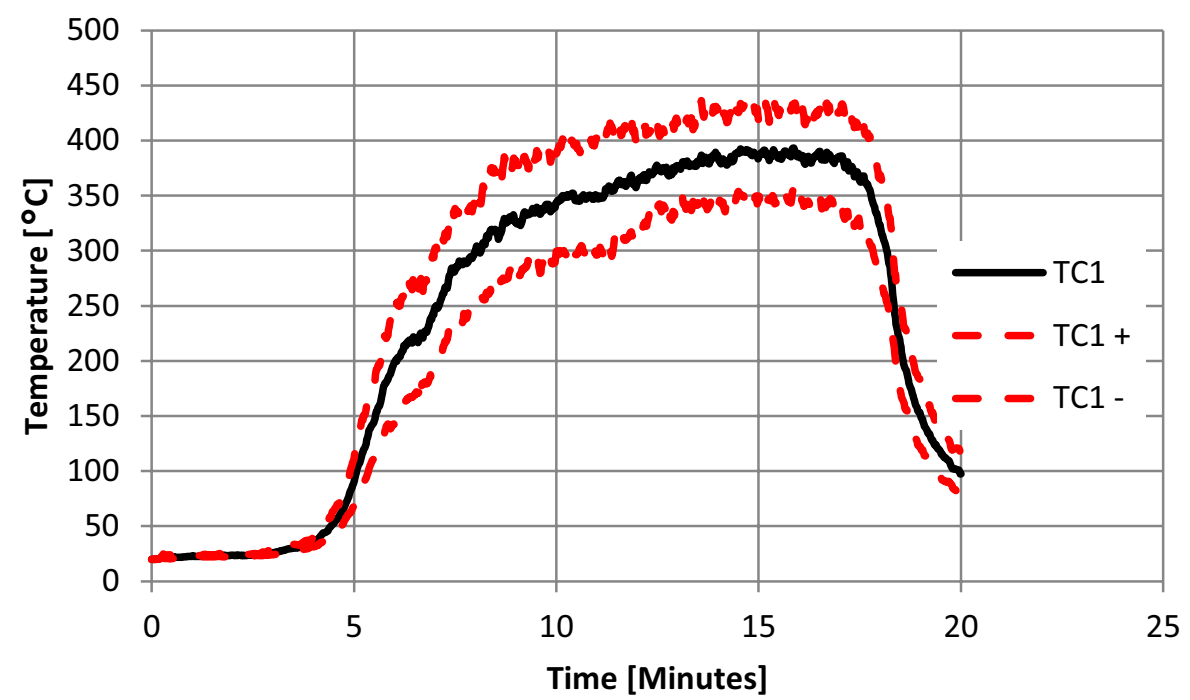

Figure 3. The temperature of TC1 (in the lower right corner of the façade) with minimum/maximum boundaries on uncertainties in the numerical simulation.

In Fig. 5, the uncertainties in the temperature at the eave will be discussed. We find a medium deviation of around $10 \%$. The heat flux computed in the middle of the first fictitious window is shown in Fig. 6 where rather large uncertainties of maximum 30\% can be observed. The determining factors for this uncertainty are the change in HRR however the number of angles in the radiation model is also very important. Note that that heat flux is the most sensitive quantity to small changes in input data and thus also from a computational point of view less practical to make comparisons with. Next we present a detailed comparison with experimental data where similarities and deviation between experimental and theoretical work will be discussed. 
$2^{\text {nd }}$ International Seminar for Fire Safety of Facades, Lund (Sweden), 2016

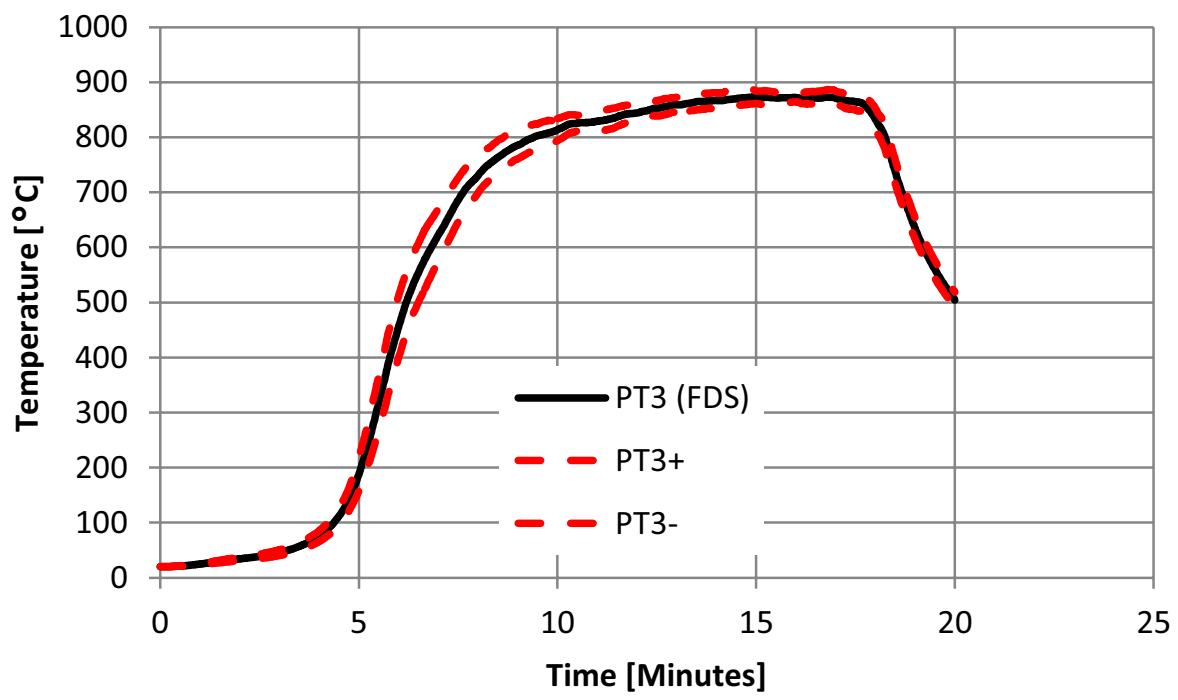

Figure 4. The temperature of PT3 (in the lower right corner of the façade) as a function of time with minimum/maximum boundaries on uncertainties in the numerical simulation.

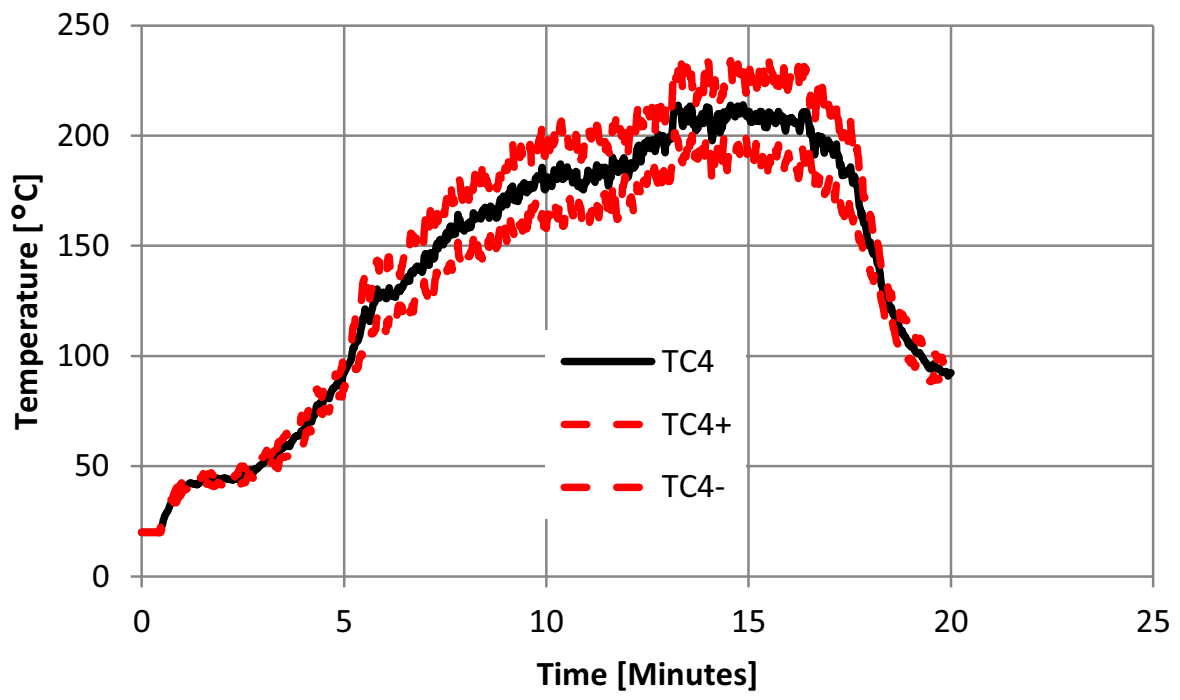

Figure 5. The temperature at TC4 (at the eave) as a function of time with minimum/maximum boundaries on uncertainties in the numerical calculation.

\section{COMPARISON WITH EXPERIMENTS}

We will here compare the measured and simulated temperatures measured by PTs in front of the façade and one just beside the heat flux gauge in the first fictitious window. It was found in Ref. [7] that varying the thickness of the façade specimen changed the heat exposure on the façade and in front of the façade; in this work we have explored this difference in the numerical model. The SP Fire 105 tests are carried out indoors in a fire testing hall, and thus the air movements are kept on a very low level. 


\section{MATEC Web of Conferences}

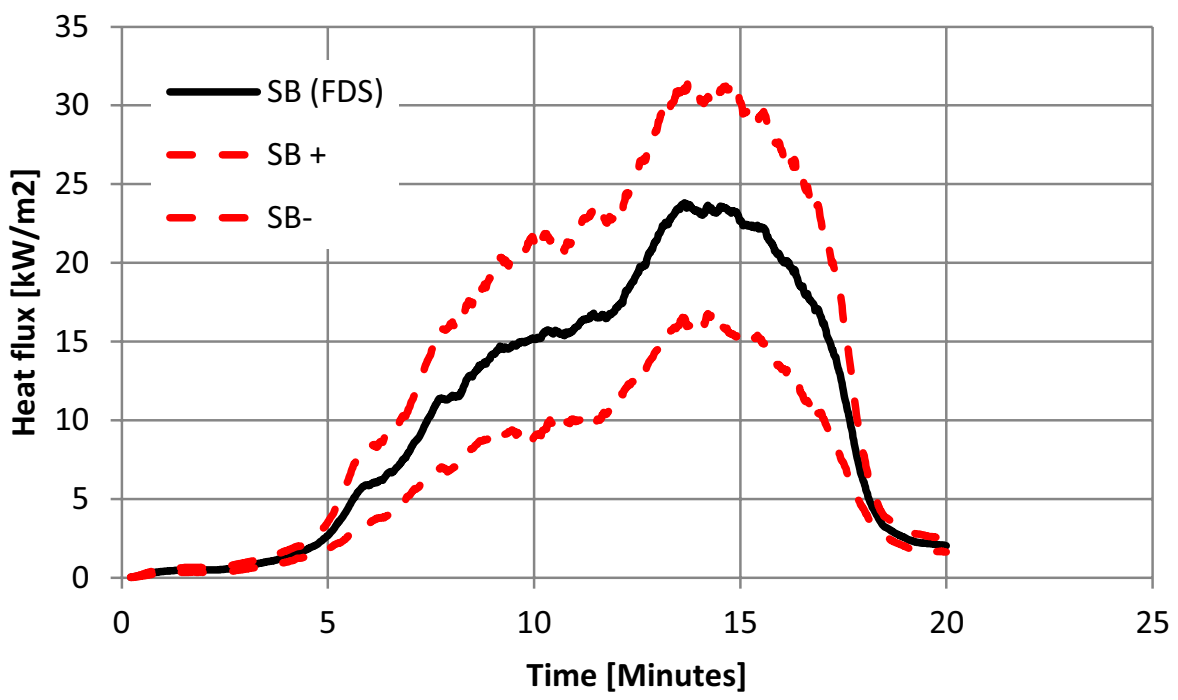

Figure 6. Heat flux (at the center of the first fictitious window) as a function of time with minimum/maximum boundaries on uncertainties in the numerical calculation (averaged over $30 \mathrm{~s}$ ).

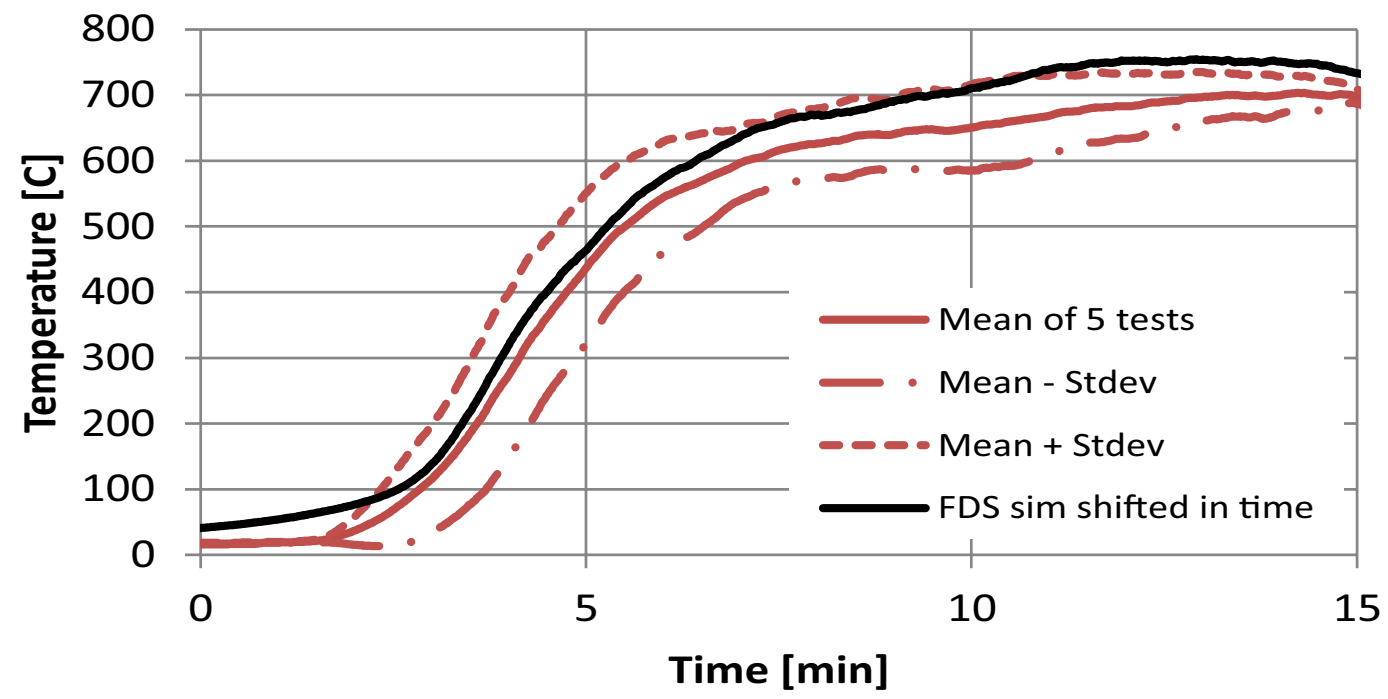

Figure 7. The resulting temperatures from the PT in front of the façade, $0.5 \mathrm{~m}$ from the fire room.

In Figs. 7 and 8 we have gauges the fire source by PTs $0.5 \mathrm{~m}$ from the fire room and one PT pointing outwards $2.1 \mathrm{~m}$ above the upper edge of the fire room (beside the heat flux meter in the fictitious window). These measurements are in addition to the standardized measurements specified in the method. The experimental data are taken from tests with three different wood with façade systems containing fire retardants, one brick wall with combustible insulation and one directly exposed insulation of phenolic resin. The data from the five tests are used to compute mean value and standard deviation and then compared to the results from the simulation. We have adjusted the time $(30 \mathrm{~s})$ for the simulation to roughly match the exposure in Fig. 7 finding a quite good qualitative and quantitative agreement 
$2^{\text {nd }}$ International Seminar for Fire Safety of Facades, Lund (Sweden), 2016

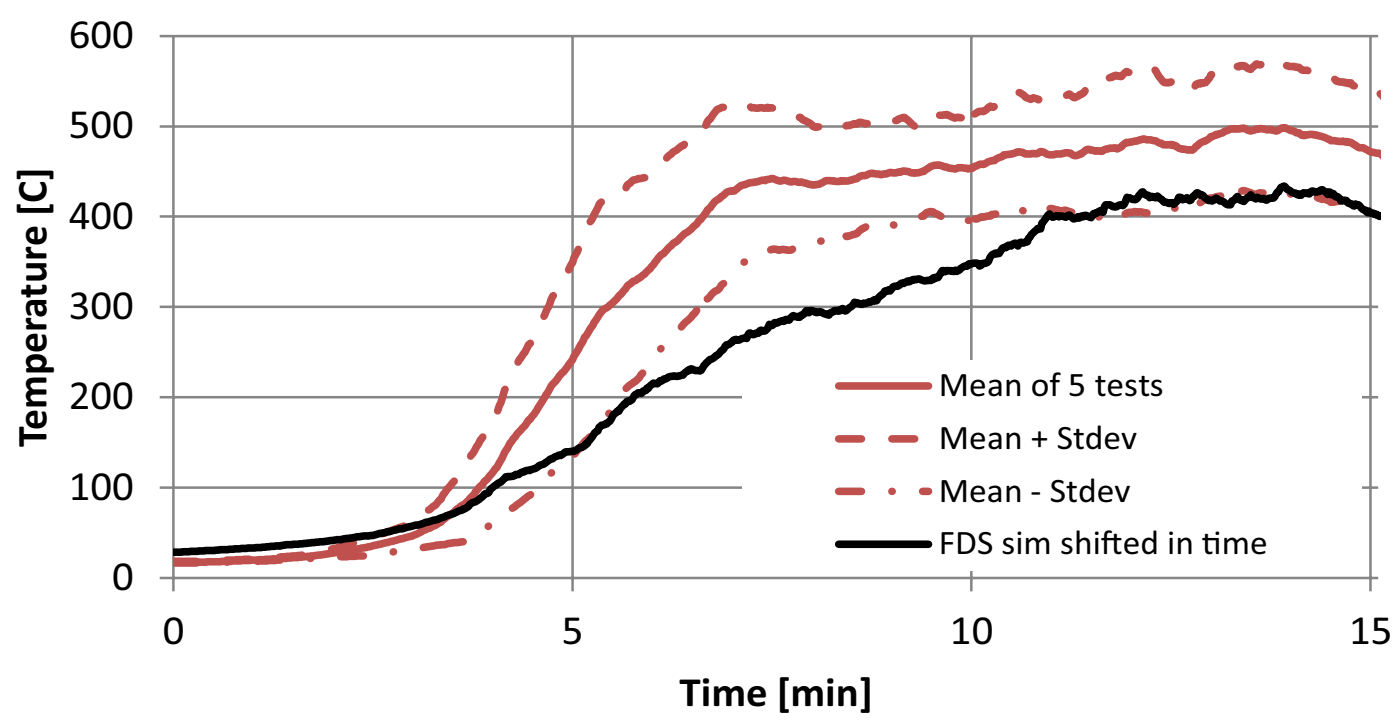

Figure 8. The resulting temperatures from the PT in the first fictitious window, $2.1 \mathrm{~m}$ above the fire room.

mostly within one standard deviation. We have approximately the same amount of uncertainty around $3 \%$ as shown Fig. 4. In Fig. 8, the exposure on the façade as estimated by a PT where we find that the temperatures in the simulation is significantly lower than those in the test however there is a significant contribution to the HRR from the wood and phenolic resin façade systems yielding increased measured temperatures.

One test deviates from the other and that is the very thick façade with bricks, see Ref. [7]. The measurements show that the plate thermometer temperature from the combustion chamber is higher than the other tests, and that the temperature $2.1 \mathrm{~m}$ above the combustion chamber is lower. This can to some extent be explained by the thickness of the façade system. The radiative heat depends on the thickness of flames. When the thickness of the façade increases, also the distance from the initial fire room to the façade surface is increased, and thus the there is a change in where the actual combustion takes place influencing the measurements by the plate thermometers. This underlines the fact that plate thermometers measure a type of effective surface temperature on a small reference specimen. This effective temperature is a much more representative quantitative measure of the thermal exposure on flat objects than the gas temperature. Furthermore the uncertainties are significantly smaller compared to the heat flux measurements. The thickness of the façade may change distance travelled by the plume, the radiation exchange with the exterior while some of the heat will be absorbed by the bottom edge, i.e. the edge facing downwards and can be seen as an extension of the fuel chamber. The thicker the specimen is, the more these factors will influence the dynamics and more heat will be absorbed by the bottom edge.

In Fig. 9 the simulated temperatures are shown for a regular façade $(100 \mathrm{~mm})$ and a thicker façade $(330 \mathrm{~mm})$ system. The same qualitative behavior as that in the experimental comparison performed in Ref. [7] where an increase temperature in front of the façade whereas a decreased temperature on the façade $2.1 \mathrm{~m}$ above the fire room is observed. Note that by varying the basic chemistry and soot yield in the simulation we can simulate the effects of varying fuel used in the fire test. We have simulated use of heptane (standard fuel), wood and propane. The results show very small differences between the different fuels on the temperatures measured by the PTs in front of the façade ( $0.5 \mathrm{~m}$ from the fire room) and the PT ( $2.1 \mathrm{~m}$ above the fire room) on the façade. 

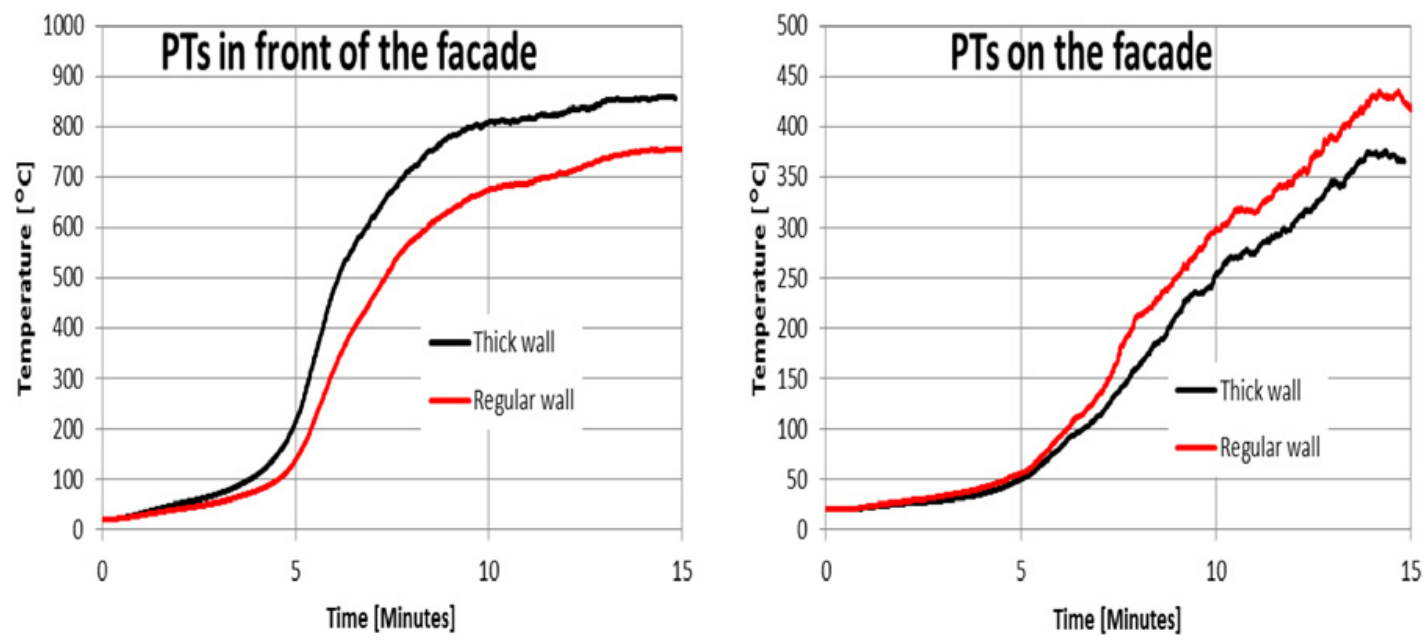

Figure 9. The results from the PT in front of the façade $(0.5 \mathrm{~m}$ from the fire room) and the PT on the facade.

Next we will investigate how these experimental and numerical results relate to similar results obtained by testing in the British standard, see the model adaptation of the façade testing method in Fig. 10. Two tests have been carried out outside Zagreb, Croatia, one in March 2014 and one in May 2014. In each test three façade rigs were used with different test specimens, i.e. three different façade systems. The three façades are prepared with different types of external thermal insulation composite systems (ETICS). Wall 1 consisted of noncombustible mineral wool insulation, Wall 2 of EPS insulation and fire stops at different heights of rock-wool insulation, and Wall 3 of EPS insulation. All three walls had an organic rendering on the surface and classified for the reaction to fire as B-s2,d0 or better. The tests were made in accordance with BS 8414-1:2002 [2], i.e. a large scale façade test method. The two tests were exactly the same, except the climate conditions since the tests were carried out outdoors. In the first test the temperature was around $15^{\circ} \mathrm{C}$ and the wind speed varied between 2 and $5 \mathrm{~m} / \mathrm{s}$. In the second test the temperature was around $25^{\circ} \mathrm{C}$ and the wind speed varied between 0 and $2 \mathrm{~m} / \mathrm{s}$. For more details on these tests see Ref. [7] where also the HRR used in the simulations are presented.

In Figs. 11 and 12 a comparison between numerical results and measured data measured on the Wall 1 with non-combustible insulation material is shown. The effect of the outdoor climate is clearly visible where the temperatures found in the measurement have large fluctuations and show consistently lower temperatures compared to the simulated values. We note that there are significant differences between the two experimental data sets sampled at different days for the same set-ups. Qualitatively similar trends are found in the simulations and experiments.

\section{DISCUSSION}

In this paper we present results from simulations and measurements concerning two large scale fire façade test methods. The numerical model representing the SP Fire 105 façade and BS 8414-1 test rigs were made in FDS however no self-consistent fire source is used but rather measured HRR are used as input. We find good repeatability between similar test set-ups however one particular test in SP Fire 105 with a thick wall stood out. In general good agreement between simulations and test results within one standard deviation are found; however there seems to be a time lag in the simulations. A similar trend with higher temperature in front of the fire room while lower temperature on the façade for the thick walled façade compared to a regular specimen was seen in both simulations and tests. In addition small 
$2^{\text {nd }}$ International Seminar for Fire Safety of Facades, Lund (Sweden), 2016
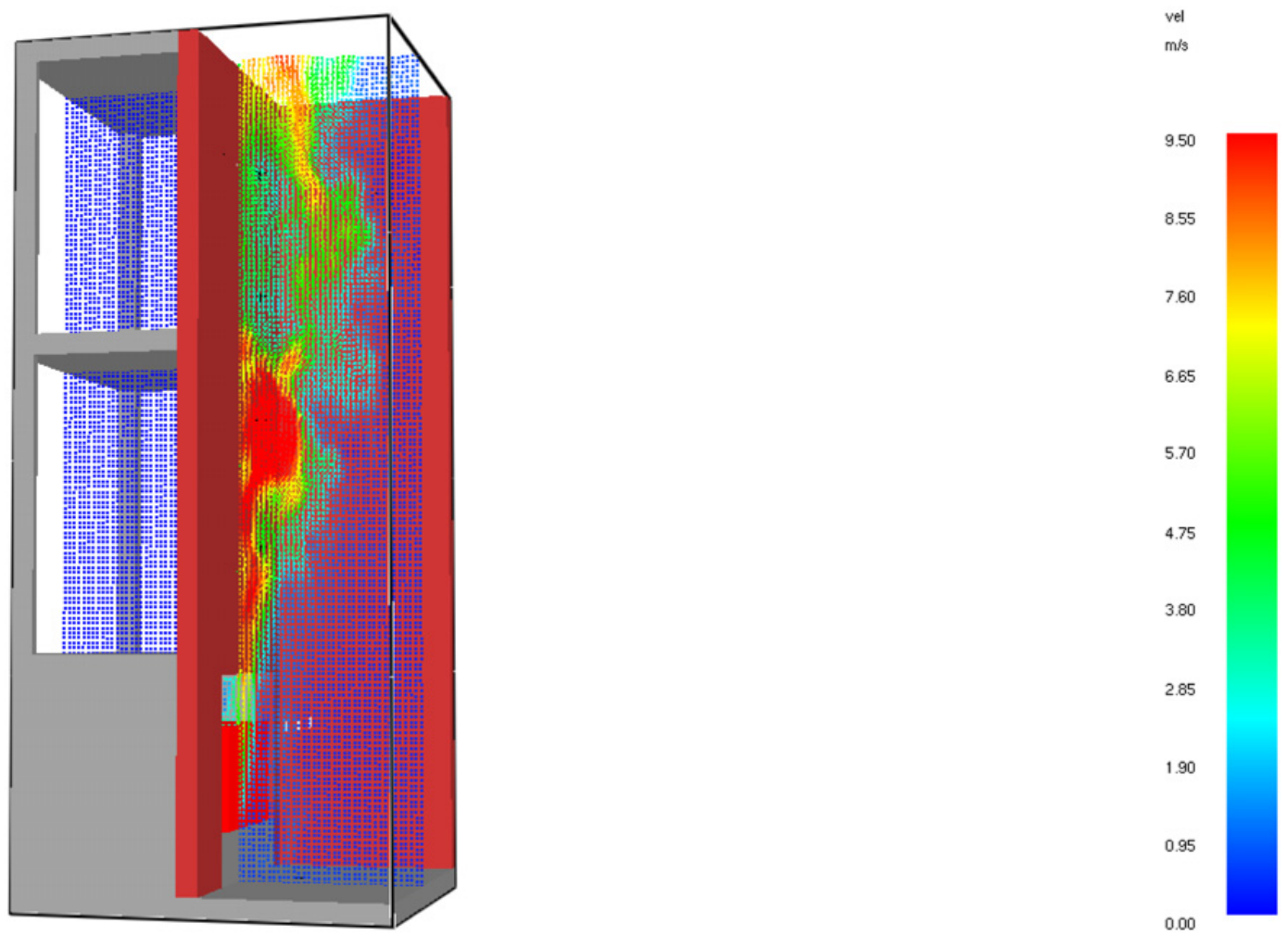

Figure 10. The model of the experimental set-up for the British standard 8414 with a velocity slice file is displayed. The positions of the PTs in front of the fire room are visible in white.

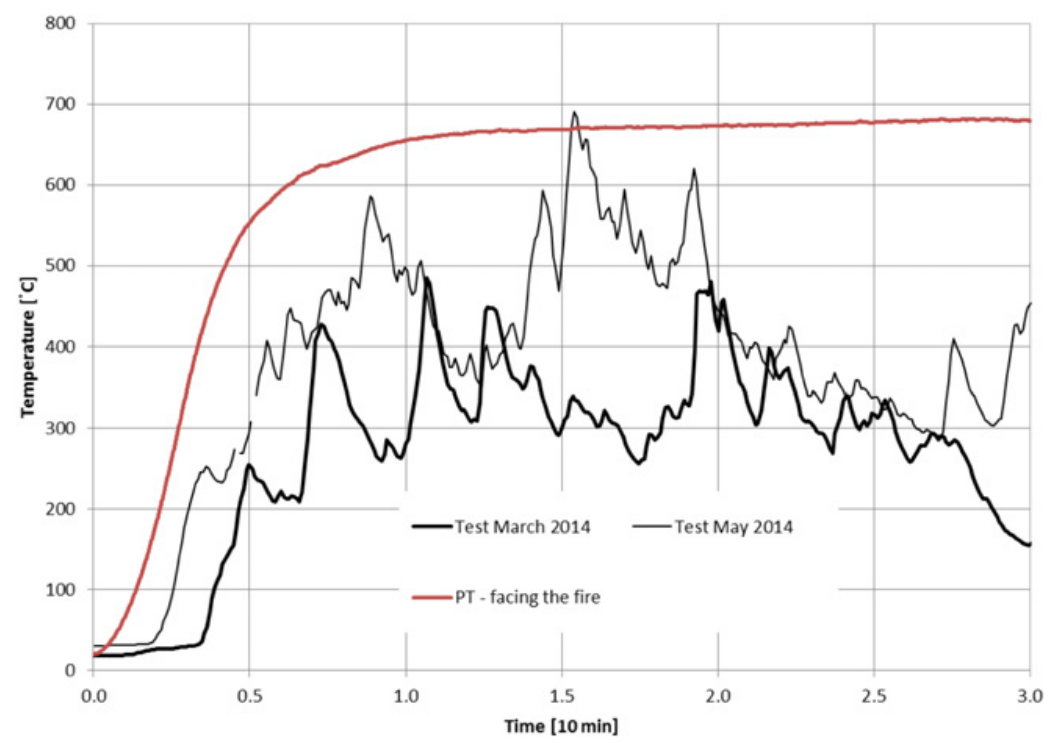

Figure 11. A comparison of temperatures measured by PTs located $0.5 \mathrm{~m}$ from the fire room in the BS8414-1 test method and simulation. 


\section{MATEC Web of Conferences}

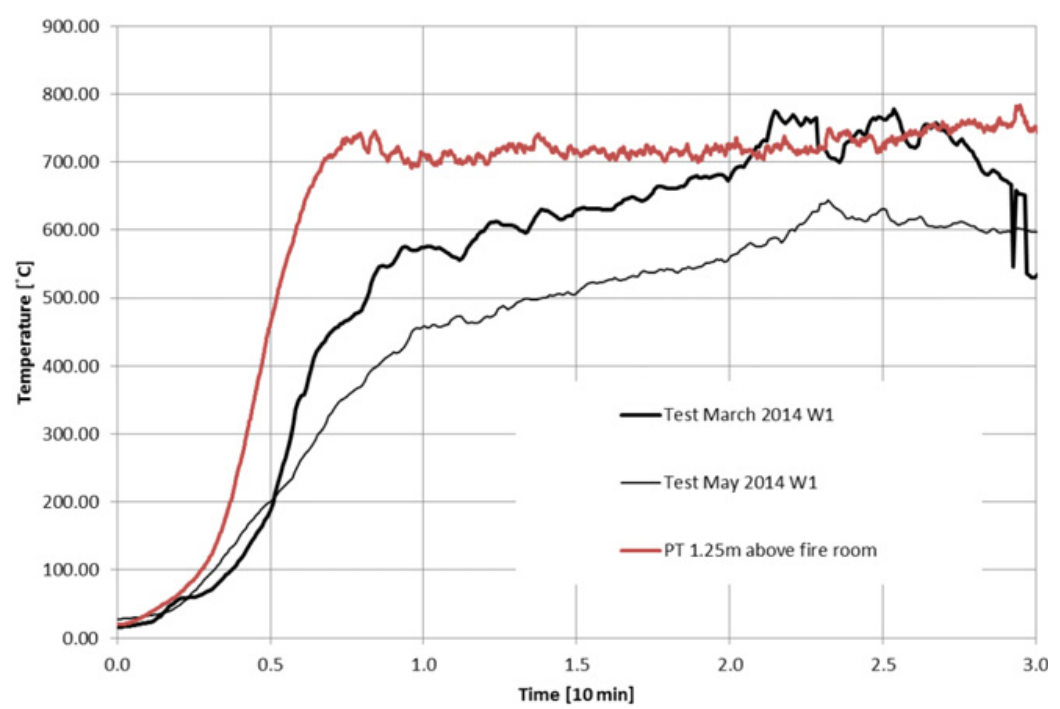

Figure 12. A comparison of temperatures measured by PTs located $1.25 \mathrm{~m}$ above the fire room in the BS8414-1 test method and simulation.

effects of varying the fuel chemistry and soot yield were seen on the computed temperatures. In order to ensure a robust, repeatable and reproducible test method for façades there are several issues that must be resolved. The heat exposure on the façade can for example be measured with plate thermometers, which is more linked with the thermal exposure of the façade surface compared to conventional thermocouples. A possible solution is to define a heat exposure curve, as in fire resistance testing, and use gas burners that can be regulated instead of using a defined amount of free burning fuel. The appropriate level and nature of the fire source has been debated for a long time and is in need of reassessment. Also a numerical tool for estimating the effects of design changes to the test methods is presented. The use of simulations may reduce the amount of large scale development testing which is inhibited by large costs.

The Authors is indebted to Dr. Hessling for constructing ensembles that made uncertainty quantification in the FDS model viable. The work presented here is sponsored by Brandforsk the Swedish Fire Research board.

\section{References}

[1] SP FIRE 105, - Method for fire testing of façade materials, Dnr 171-79-360 Department of Fire Technology, Swedish National Testing and Research Institute.

[2] British Standard BS 8414-1:2002, Fire performance of external cladding systems - Part 1: Test method for non-loadbearing external cladding systems applied to the face of the building (2002).

[3] White, N. and Delichatsios, M., Fire Hazards of Exterior Wall Assemblies Containing Combustable Components, National Fire Protection Association (NFPA) Fire Protection Research Foundation Report (2014).

[4] Yokoi, S. Study on the prevention of fire-spread caused by hot upward current. Japanese Ministry of Construction, Building Research Institute Report 34, 1960.

[5] Ödeen K. \& Nordström Å., Fire and smoke spread along facades and in ventilation ducts (in Swedish), Arbetshandling 12:1967 från Byggforskningen, Statens Provningsanstalt (SP Technical Research Institute of Sweden), 1967. 
[6] Ondrus, J. and Pettersson, O., Fire hazards of façades with externally applied additional thermal insulation, LUTVDG/(TVBB-3025) (1986).

[7] Anderson, J., Boström, L., Jansson, R., Milovanović, B., Fire dynamics in façade fire tests: measurement, modelling and repeatability, Applications in Structural Fire Engineering, 15-16 October 2015.

[8] Anderson, J. and Jansson, R., Façade fire tests - measurements and modeling, MATEC Web of Conferences 9, 02003 (2013).

[9] Anderson, J., Jansson, R., Fire Dynamics in Façade Fire Tests: Measurement and Modeling, Proceedings of Interflam 2013, page 93, Royal Holloway College, University of London UK, (2013).

[10] Jansson, R., Anderson, J., Experimental and numerical investigation of fire dynamics in a façade test rig, Proceedings of Fire Computer Modeling, Santander, Spain, 18-19th October 2012, p. 247.

[11] McGrattan, K., McDermott, R. Hostikka, S. Floyd, J., Fire Dynamics Simulator (Version 6) user's guide. NIST Special Publication 1019, 2015.

[12] Guide for the Verification and Validation of Computational Fluid Dynamics Simulations (AIAA G-077-1998 (2002)).

[13] Quality Management in Engineering Simulation, A Primer for NAFEMS QSS, NAFEMS 2014.

[14] Hessling, J.P., Deterministic sampling of propagating model covariance, SIAM/ASA J. Uncertainty Quantification 1, pp 297-318 (2013).

[15] Hermansson, K., Quality assurance and the simulation of fires - A practical application for automated validation of user-generated input data for FDS, Thesis Report 5501 Lund University (2015).

[16] Babrauskas, V., Facade Fire Tests: Towards an International Test Standard, Fire Technology 32, 219-230 (1996). 